
\title{
25 Research Square \\ Effects of Vitamin D Supplementation on Female Vitamin D Deficient Teenagers: A Randomized Clinical Trial
}

Salma Ahi ( $\sim$ salmaahi.61@gmail.com)

Jahrom University of Medical Science

Fatemeh Gholami

Jahrom University of Medical Science

Naser Hatami

Jahrom University of Medical Science

Fatemeh Golabi

Jahrom University of Medical Science

Research article

Keywords: Vitamin D Deficiency, Anthropometric Indicators, female adolescents.

Posted Date: July 15th, 2020

DOI: https://doi.org/10.21203/rs.3.rs-33594/v1

License: (c) (i) This work is licensed under a Creative Commons Attribution 4.0 International License.

Read Full License 


\section{Abstract}

Background: Vitamin D deficiency is one of the most prevalent disorders worldwide. Considering the magnitude of growth in adolescence, the aim of this study was to ascertain the prevalence of vitamin D deficiency and discover the association between anthropometric indices and vitamin $D$ levels and the effect of vitamin D treatment on anthropometric indices in adolescent girls.

Method: we performed this randomized clinical trial study (approved by Iranian Registry of Clinical Trials with code of IRCT20200615047785N2) conducting 313 high school girl students in a random cluster sampling from Jahrom city schools. Blood samples were collected from participants to determine the serum level $(\mathrm{OH})$ D25. We divided vitamin D deficient patients into two groups: control who received placebo $(n=150)$ and intervention group $(n=150)$ who were prescribed one tablet of 50,000 units of vitamin D per week for eight weeks. Anthropometric indices of participants were measured before and after the intervention. Datas were analyzed by SPSS software version 19.

Results: The prevalence of vitamin D deficiency was $95 \%$. Body mass index had no significant difference before the intervention in both groups $(P=0.76)$. After intervention in the case group, body mass index increased significantly $(P=0.01)$, but there was no significant difference in the control group after treatment $(P=0.42)$. There was no significant difference in waist circumference, height and weight before and after the intervention in both groups $(P>0.05)$. There was also a weak but significant correlation between height, weight, and baseline $250 \mathrm{HD}$ levels of participants.

Conclusion: The results of this study showed that there is a reverse and significant relationship between height and weight index with 250HD. Further monitoring and prolonged studies with extended follow ups might improve the anthropometric parameters after treatment.

\section{Background}

Vitamin $D$ is a prohormone that is mainly produced by Sunlight exposure to the skin, although it can be achieved from food and supplements that stand for less than $10 \%$ of body vitamin D level. (1). Vitamin D is an essential component for healthy bone metabolism, growth, and development. various metabolic processes require vitamin $D(2)$, as it increases the absorption of phosphorus and calcium from the intestines and reduces their excretion from the kidney and diminishes the osteoporosis process.

Therefore, vitamin $D$ deficiency is one of the critical factors in the development of bone metabolism disorders (3). Vitamin D deficiency is one of the most critical factors in bone metabolism disorders. Also, vitamin $D$ receptors have been observed in many other organs, which indicates that vitamin $D$ deficiency causes several complications in various organs. The prevalence of vitamin D deficiency in Iran is $75.1 \%$ for women and $72.1 \%$ for men, approximately (4). There are several types of vitamin D supplements to treat this disorder, including ergocalciferol (vitamin D2 ) and colo-calciferol(vitamin D3) (5). Anthropometry word is derived from the two Greek words, "Anthropos" means human, and "Metri" means measurement. It is a science that measures body dimensions, including some factors like the height, 
weight, the field of motion and the strength of the muscles of the body(6). Anthropometrics examines items such as BMI, Waist circumflex. Most studies have described the significant associations of vitamin D levels and anthropometric indices (7-9). However, it has been revealed that vitamin D treatment is not able to improve some anthropometric indices like height (10). Respecting the different results found in previous studies, we aimed to determine the effect of vitamin $D$ treatment on anthropometric indices in vitamin $\mathrm{D}$ deficient female adolescents.

\section{Method}

\section{Study population}

The present study was a randomized clinical trial by approved by Iranian Registry of Clinical Trials with code of IRCT20200615047785N2 at 2020-06-25. We included a total of 412 patients at the baseline. After excluding 16 participants who had a history of Diabetes, growth hormone consumption, recipients of vitamin $D$, celiac disease and participants who had a metabolic bone disease, 396 girls from different schools in Jahrom were studied. Eventually, we diagnosed 313 people with vitamin D deficiency. 13 participants were also excluded because of lost follow ups. One hundred fifty patients were treated in the case group. One hundred fifty patients were included in the control group which received placebo during the study period; but finally they completed vitamin D treatment at the end of the study, considering the ethical issue.

Serum 250HD levels of all participants were measured during the October after sufficient sun exposure during the summer. Blood samples were taken from 8 am to $9 \mathrm{am}$. Serum level 250HD levels were assayed with ELC technique. In this study, vitamin D sufficient participants defined with serum $250 \mathrm{HD}$ levels above $32 \mathrm{ng} / \mathrm{ml}$; vitamin D insufficient subjects characterized with serum $250 \mathrm{H}$ D levels between 15 and 32ng/ml; vitamin D deficiency defined as drum $250 \mathrm{HD}$ levels lower than $15 \mathrm{ng} / \mathrm{ml}$.

Participants were randomly assigned to two groups; the first group was treated with vitamin D (case group) and the control group (non-treatment). The case group was treated with a total dose of $50000 \mathrm{IU}$ per week for 8 weeks (Zahravi $®$ Company). Because of increased growth velocity in adolescents, the importance of vitamin $D$ supplementation in this population is the most. After the completion of the course, students were again evaluated for anthropometric indices.

To reduce the measurement error, two experts measured the height, weight, and waist circumference with standard wall mounts, standard scales and tape measurements in two steps before and after treatment. The waist circumference was determined as the distance between the Pubis Symphysis and sternum in the umbilical line and above the iliac crest. All participants wear light clothes and didn't wear shoes or hair clips at the measurement time. To eliminate the confounding factors, both participants and operators were not comprehended of vitamin D levels or treatment groups.

\section{Statistical Analysis}


Descriptive statistics indexes of mean and standard deviation, percentage, median and inter-quartile range were used to describe participants' information, and analytical statistics (Mann-Whitney, Wilcoxon and Spearman correlation) were used at a significant level of 0.05, for comparison by SPSS version 21 software.

\section{Results}

According to Table 1, the results showed that there were no significant difference between the two groups in terms of quantity of variables such as age, weight, height, waist circumference, and BMI before the intervention $(P>0.05)$, so it can be interpreted that the alignment between the two groups is well done. Waist, height, and weight indices were not significantly different between the two groups after intervention ( $P>0.05)$, which indicates that the intervention was not effective on waist circumference, height, and weight. There were significant differences in Waist circumflex, height and weight indices between the before and after the intervention in both study groups $(P<0.05)$. However, these observed differences may not be attributed to vitamin $D$ purely because this variation happened in both groups.

Table 1

Study variables pre and post treatment with Vitamin D

\begin{tabular}{|c|c|c|c|c|}
\hline \multirow{2}{*}{\multicolumn{2}{|c|}{ variable }} & Case group & Control group & p-value \\
\hline & & & & Mann-Whitney U \\
\hline \multicolumn{2}{|l|}{ Age } & $11.62 \pm 0.49$ & $11.56 \pm 0.49$ & 0.8 \\
\hline \multicolumn{2}{|c|}{ Vitamin D, ng/ml } & 4.2 & 4.8 & 0.1 \\
\hline \multirow[t]{2}{*}{$\mathrm{BMI}, \mathrm{kg} / \mathrm{m} 2$} & Pre-treatment & 19.20 & 19.54 & 0.76 \\
\hline & Post-treatment & $19.46^{\star}$ & 19.61 & 0.69 \\
\hline \multirow[t]{2}{*}{ Waist, cm } & Pre-treatment & 77 & 76 & 0.5 \\
\hline & Post-treatment & $75.50 *$ & $78.0 *$ & 0.61 \\
\hline \multirow[t]{2}{*}{ Height, cm } & Pre-treatment & 150 & 148.75 & 0.51 \\
\hline & Post-treatment & $151^{*}$ & $151.5^{\star}$ & 0.56 \\
\hline \multirow[t]{2}{*}{ Weight, kg } & Pre-treatment & 43.70 & 43.90 & 0.74 \\
\hline & Post-treatment & $41.10 *$ & 45 & 0.61 \\
\hline
\end{tabular}

Besides, there was no significant difference in BMI of both case and control groups after intervention ( $p=$ $0.69)$; but the significant difference between before and after intervention was observed in the cases ( $p<$ 0.05); according to not significant difference in controls; the role of vitamin D was highlighted in this difference. 
A reverse significant relationship between height and $250 \mathrm{HD}$ levels before and after treatment was observed ( $p=0.001, r=-0.353)$. Also the reverse significant relationship was observed between pretreatment serum $250 \mathrm{HD}$ levels and weight $(p=0.001, r=-0.205)$. No significant correlation was observed for other parameters.

\section{Discussion}

Prolonged Vitamin D deficiency leads to metabolic bone diseases like osteoporosis and osteomalacia, its role in autoimmune diseases such as type 1diabetes mellitus(11), SLE (12), multiple sclerosis (13) and malignancy has been confirmed (14). Vitamin D enrich diet is necessary whenever sun exposure is not adequate (15). So vitamin D fortified foods have been used in different countries yearly $(16,17)$. Because of higher rate of growth velocity in youth, their micronutrients requirement such as vitamin $D$ is higher too. A routine balanced diet may provide all necessitated nutrients except vitamin $\mathrm{D}$.

Iranian diet has no significant source of vitamin D and food products didn't fortified with vitamin D (18). Hence, the role of vitamin D in growth of Iranian teens hasn't been explored, this study was conducted to investigate the efficacy of vitamin D supplements on anthropometric indices in sixth grade school girls of Jahrom ; a city located at south west of Fars province.

The results showed that the prevalence of vitamin D deficiency among participants was $95 \%$, which is higher than previous studies. Saki et al. (19), Alizadeh et al.(20) and Faraji et al. (21) also reported the prevalence of vitamin deficiency in their study as $81.3 \%, 70 \%$ and $68 \%$ respectively indicating a high prevalence of vitamin D deficiency in this age range. Even though Jahrom is located in the south west of Iran where the weather is almost always warm and sunny ,blood sampling for this study was performed in September and October to reduce the bias of low sun exposure in winter,nevertheless the prevalence of vitamin D deficiency was still high. Khashayar et al. (22) also observed that there is a weak linear relationship between the increase in body mass index and vitamin $D$ levels. Another study, based on data from 21 cohort studies with 42024 adult participants by Vimaleswaran et al., revealed that an increase in $\mathrm{BMI}$ is associated with an increased incidence of vitamin $\mathrm{D}$ deficiency, but a low level of vitamin $\mathrm{D}$ has little effect on an increase in body mass index (23).

However, contrary to the results of this study, Motlaghzadeh et al.(24) observed that serum vitamin D level had a significant correlation with body mass index before and after treatment. Sakki et al. also observed that serum levels of $(\mathrm{OH}) \mathrm{D} 25$ have a significant reverse correlation with body mass index and puberty, and exposure to sunlight has a significant direct relationship (18). Najarzadeh et al. (25) also did not explain significant differences in the anthropometric indices after 3 months of supplementation with vitamin D (mean BMI, BMI, waist to hip ratio). Bonakdaranet al. also perceived that vitamin D deficient patients had a clear difference in body mass index $(p=0.003)$ compared to patients with normal levels of vitamin D. in other words, There is a reverse and significant relationship between serum vitamin $D$ levels and body mass index(26). In the study of McGill et al., There was a significant and inverse relationship between the weight, body mass index and abdominal circumference (27). 
However, in the study of Geng $S$ et al.(28), there was no significant relationship between the levels of $(\mathrm{OH})$ D 25 and the anthropometric indexes (body mass index). Khor et al. observed that there was a significant correlation between serum vitamin $D$ level and body mass index $(p=.16)$ among boys $(p=.16)(9)$.

However, according to cross sectional studies' limitations, confirmation of cause and effect is not clear. Due to obesity, vitamin $D$ is absorbed and seized in excess adipose tissue which causes decreasing the availability of vitamin $D$, leading to reduction in serum vitamin $D$ levels. On the other hand, obesity and vitamin $D$ deficiency can both happen due to low exposure to sunlight due to the lack of physical activity outside of the home. Another hypothesis is that vitamin D deficiency increases the risk of becoming overweight.

It was also observed that there was no significant difference in waist circumference between the two groups after intervention $(P=0.6)$. Significant differences in waist circumferences of cases and controls were observed before and after intervention $(P=0.001)$. However, since observed differences remained significant in both groups, it cannot be attributed to the effect of vitamin $D$.

Najarzadeh et al. also observed that the mean waist circumference in the treatment group was significantly decreased throughout the study period $(p=0.05)(24)$. In another study, Faraji et al. also found that there are a reverse and significant relationship between serum vitamin $D$ levels with waist circumference $(p<0.02)$, height $(p<0.001)$ and Waist-hip ratio $(p<0.007)$, but has not significantly changed with hip circumference and BMI variables(20). The results of Al-Mulhim et al. study, showed that waist circumference in the intervention group significantly decreased compared to the pre-treatment group However, no significant differences were observed in other anthropometric indices after intervention. Vitamin D supplementation decreased women's waist circumference significantly while not affecting other anthropometric indices (29).

N. Phetkrajaysang et al. also observed that increased waist circumference could increase the risk of vitamin D deficiency (7). In another study, GILBERT-DIAMOND et al. showed that serum vitamin D levels have a significant and reverse relationship with the waist circumference and body mass index. Thus, it can be concluded that the serum level of vitamin $D$ has a significant and reverse relationship with obesity in children at school age (10). In another study, Gonca Tamer et al. showed that body mass indexes, waist circumference, and hip circumference, the rate of obesity and abdominal obesity were significantly lower in vitamin D sufficient subjects. It was also observed that the Serum $(\mathrm{OH}) \mathrm{D} 25$ level had significant reverse relationship with body mass index $(r=-0.481, p<0.0001)$, waist circumference $(r=-0.480, p<$ 0.0001), and Waist-hip ratio (8).

Other results of the present study showed that there is a significant and reverse relationship between height and serum vitamin D levels $(r=-0.266)$, and also the height index after intervention in the two groups did not significantly change $(P=0.55)$, which indicates that the intervention was not effective during this time interval on the height index. Also, intra-group comparisons showed a significant difference between measured height before and after the treatment in both groups $(p=0.001)$ which as we mentioned, it cannot be attributed to vitamin $\mathrm{D}$. 
Khalaji et al. observed that serum levels of D3 $(\mathrm{OH}) 25$ had a significant and direct relation with exposure to sunlight, but had a significant and reverse relation with weight, height, and body mass index. Therefore, the high prevalence of vitamin D deficiency in Primary school children (especially girls) requires urgent intervention and appropriate nutritional support (30). Other results of this study also showed that the prevalence of vitamin D deficiency among tall children is about $61 \%$. Considering the short stature growth disorder in the case group compared to the control group, it seems that the eight weeks follow up is not sufficient for evaluation of vitamin D effects; so we suggest further studies with prolonged follow ups for better observation.

Other results of the present study showed that there is a reverse, and significant relationship between weight and serum vitamin $D$ levels $(r=-0.205$ and $p=0.001)$, and also the weight index after intervention in both groups was significantly different $(P=0.61)$, which indicates that the intervention is not affected the weight. There is a significant difference in measured weight between before and after the treatment results in the control group $(P=0.001)$. Also in the case group, the difference in weight was significant before and after treatment $(P=0.001)$. So the observed difference cannot be as a result of vitamin $D$ treatment. To consider the ethical issue, after the study time, the control group received the vitamin $\mathrm{d}$ supplement as it was helpful.

E Rodríguez-Rodríguez et al. also showed that only weight and body mass index could be dependent on the level of vitamin D, and it was also observed that children with higher BMI, weight, and waist circumference have a higher risk of vitamin D deficiency. Therefore, it can be concluded that BMI and abdominal obesity have a significant effect on vitamin D deficiency in children (31).

\section{Conclusion}

The results of this study showed that height and weight indices have reverse and significant relationship with vitamin D. Prolonged monitoring and evaluation is recommended to better observe vitamin $D$ effects on teenagers anthropometric indices.

\section{Abbreviations}

Body mass index (BMI); Systemic lupus erythematosus (SLE)

\section{Declarations}

Ethics approval and consent to participate: this study was approved by the ethics committee of Jahrom University of medical sciences (with registration code of IR.JUMS.REC.1394.162). All participants and their parents filled written informed consent form.

Consent to publish: there was no personal identifying data in this manuscript.

Availability of data and materials: There isn't any additional data. 
Competing interests: None.

Funding: We didn't received any grant from funding agencies.

Authors' Contribution: SA had designed the study and sample collecting was done by SA. NH conducted data analysis. GoF and GhF have written the draft manuscript and all authors have contributed in reversions. All Authors read and approved the manuscript.

Acknowledgments: We would like to thank the Clinical Research Development Unit of Peymanieh Educational and research and therapeutic Center of Jahrom University of medical sciences for providing facilities for this work.

\section{References}

1. Misra M, Pacaud D, Petryk A, et al. Vitamin D deficiency in children and its management: review of current knowledge and recommendations. Pediatrics. 2008;122:398.

2. BrownAJ,DussoA,SlatopolskyE.Vitamin DAm. J Physiol. 1999;277(2pt2):F157-75.

3. Holick MF, Vitamin D. importance in the prevention of cancers, type 1 diabetes, heart disease, and osteoporosis. Am J Clin Nutr. 2004;79(3):362-71.

4. Moradzadeh K, Larijani B, Keshtkar AA, Hossein-Nezhad A, Rajabian R, Nabipour I, Omrani GH, Bahrami A, Gooya MM, Delavari A. Normative values of vitamin D among Iranian population: a population based study. International Journal of Osteoporosis \& Metaboli.

5. Bolland MJ, Grey A, Gamble GD, Reid IR. Calcium and vitamin D supplements and health outcomes: a reanalysis of the Women's Health Initiative (WHI) limited-access data set. The American journal of clinical nutrition. 2011 Aug 31;94(4):1144-9.

6. Elshennawy AK, Lee,C.H, Hines ,M,1989,Ergonomics Issues in Quality control computers Ind., Engng. 17(1-4)514-518.

7. Phetkrajaysang N, Sansanayudh N, Wongwiwatthananukit S, et al. (2017). Original article. Prevalence of vitamin $D$ deficiency and association of serum vitamin D level with anthropometric and metabolic factors in metabolic syndrome patients. Asian Biom.

8. Tamer G, Mesci B, Tamer I, Kilic D, Arik S. Is vitamin D deficiency an independent risk factor for obesity and abdominal obesity in women? Endokrynol Pol. 2012;63(3):196-201.

9. Khor, et al. High prevalence of vitamin D insufficiency and its association with BMI-for-age among primary school children in Kuala Lumpur, Malaysia. BMC Public Health. 2011;11:95.

10. Razzaghy-Azar M, Shakiba M. Assessment of vitamin D status in healthy children and adolescents living in Tehran and its relation to iPTH, gender, weight and height. Annals of human biology. 2010 Oct 1;37(5):692-701.

11. Zipitis CS, Akobeng AK. Vitamin D supplementation in early childhood and risk of type1 diabetes: a systematic review and meta-analysis. Arch Dis Child. 2008;93:512-7. 
12. Amital H, Szekanecz Z, Szücs G, Dankó K, Nagy E, Csépány T,et al. Serum concentrations of 25-OH vitamin D in patients with systemic lupus erythematosus (SLE) are inversely related to disease activity: is it time to routinely supplement patients with SLE w.

13. Ascherio A, Munger KL, Simon KC. Vitamin D and multiple sclerosis. Lancet Neurol(. 2010;9:599612.

14. Trump DL, Deeb KK, Johnson CS. Vitamin D: considerations in the continued development as an agent for cancer prevention and therapy. Cancer. 2010;16:1-9.

15. Bischoff-Ferrari HA, Giovannucci E, Willett WC, et al. Estimation of optimal serum concentrations of 25-hydroxyvitamin D for multiple health outcomes. Am J Clin Nutr. 2006;84:18-28.

16. Keane EM, Healy M, O’Moore R. Vitamin Dfortified liquid milk: benefits for the elderly communitybased population. Calcif Tissue Int. 1998;62:300-2.

17. Keane EM, Rochfort A, Cox J, et al. Vitamin-Dfortified liquid milk-a highly effective method of vitamin D administration for house-bound and institutionalised elderly. Gerontology. 1992;38:280-4.

18. Neyestani TR, Hajifaraji M, Omidvar N, Eshraghian MR, Shariatzadeh N, Kalayi A, Gharavi AA, Khalaji $\mathrm{N}$, Haidari H, Zowghi T, Nikooyeh B. High prevalence of vitamin D deficiency in school-age children in Tehran, 2008: a red alert. Public health nutrition. 2.

19. Saki F, Dabbaghmanesh M, Omrani G, Bakhshayeshkaram M. Vitamin D deficiency and its associated risk factors in children and adolescents in southern Iran. Public Health Nutrition. 2017;20(10):1851-6. doi:10.1017/S1368980015001925.

20. Alizadeh Z, Halabchi F, Rajabian Tabesh M. Vitamin D deficiency in population with BMI greater than $25 \mathrm{~kg} / \mathrm{m} 2$ and its association with demographic and anthropometric indices. Tehran Univ Med J. 2015;73(6):447-55.

21. Faraji R, Sharami SH, Zahiri Z, Asgharni M, Kazemnejad E, Sadeghi S. Evaluation of Relation between Anthropometric Indices and Vitamin D Concentrations in Women with Polycystic Ovarian Syndrome. Journal of Family Reproductive Health. 2014;8(3):123-9.

22. Khashayar P, Meybodi HR, Soltani A, Taheri E, Homami MR, Heshmat R, et al. Association between vitamin D levels and BMI values in an Iranian population. Clin Lab. 2014;60(3):383-9.

23. Vimaleswaran KS, Berry DJ, Lu C, Tikkanen E, Pilz S, Hiraki LT, et al. Causal relationship between obesity and vitamin D status: bidirectional Mendelian randomization analysis of multiple cohorts. PLoS Med. 2013;10(2):e1001383.

24. Yasaman Motlaghzadeh F, Sayarifard B, Allahverdi A, Rabbani A, Setoodeh A, Sayarifard. Farzaneh Abbasi, Mohammad-Taghi Haghi-Ashtiani, Abbas Rahimi-Froushani; Assessment of Vitamin D Status and Response to Vitamin D3 in Obese and Non-O.

25. ON ANTHROPOMETRIC PARAMETERS IN PATIENTS WITH METABOLIC SYNDROME. TOLOO-EBEHDASHT JUNE-JULY

Najarzadeh A, Esteghamati A, Fallahzadeh H, Saeedi E, EFFECT OF VITAMIN D SUPPLEMENTATION. ON ANTHROPOMETRIC PARAMETERS IN PATIENTS WITH METABOLIC SYNDROME. TOLOO-EBEHDASHT JUNE-JULY 2014, Volume 13, Number 2 (44); Page(s) 25 To 36. 
26. Bonakdaran S, Varasteh A, Khaajeh-Dalouie M. Serum 25 Hydroxy Vitamin D3 and Laboratory Risk Markers of Cardiovascular Diseases in Type 2 Diabetic Patients. Iranian Journal of Endocrinology Metabolism. 2010;11(5):504-9.

27. Mc Gill AT, Stewart JM, Lithander FE, Strik CM, Poppitt SD. Relationships of low serum vitamin D3 with anthropometry and markers of the metabolic syndrome and diabetes in over weight and obesity. Nutr J. 2008 Jan 28; 7: 4.

28. Geng SS, Ma JQ, Liu SS, Zhang J, Sheng XY. Vitamin D Insufficiency and Its Association with Biochemical and Anthropometric Variables of Young Children in Rural Southwestern China. Chin Med J. 2016;129:1273-9.

29. Al-Mulhim NS, Eldin TG, Latif R, Al-Asoom LI, Al-Sunni A. Effects of vitamin D supplementation on anthropometric indices in vitamin D-deficient obese Saudi females; a randomized controlled trial. Saudi J Health Sci. 2015;4:83-7.

30. Khalaji N, Asadzadeh S, Neyestani T, Hajifaraji M, Omidvar N, Shariatzadeh N, et al. High prevalence of vitamin D deficiency in school age children in Tehran, 2008: a red alert. Iranian Journal of Nutrition Sciences Food Technology. 2013;7(5)::389-39.

31. Rodríguez-Rodríguez E, Navia-Lombán B, López-Sobaler AM, Ortega RM. Associations between abdominal fat and body mass index on vitamin D status in a group of Spanish schoolchildren.

\section{Supplementary Files}

This is a list of supplementary files associated with this preprint. Click to download.

- CONSORT2010Checklist1.doc 